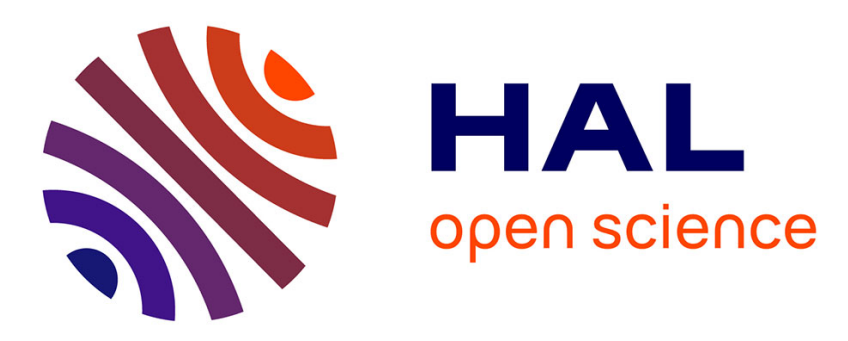

\title{
Parameters and states estimation for Dengue epidemic model
}

\author{
Ramdane Tami, Driss Boutat, Gang Zheng, Frédéric Kratz
}

\section{To cite this version:}

Ramdane Tami, Driss Boutat, Gang Zheng, Frédéric Kratz. Parameters and states estimation for Dengue epidemic model. 13th European Control Conference, Jun 2014, Strasbourg, France. hal01094912

\section{HAL Id: hal-01094912 \\ https://hal.inria.fr/hal-01094912}

Submitted on 14 Dec 2014

HAL is a multi-disciplinary open access archive for the deposit and dissemination of scientific research documents, whether they are published or not. The documents may come from teaching and research institutions in France or abroad, or from public or private research centers.
L'archive ouverte pluridisciplinaire HAL, est destinée au dépôt et à la diffusion de documents scientifiques de niveau recherche, publiés ou non, émanant des établissements d'enseignement et de recherche français ou étrangers, des laboratoires publics ou privés. 


\title{
Parameters and states estimation for Dengue epidemic model
}

\author{
Ramdane Tami $^{*, 1}$, Driss Boutat ${ }^{1}$, Gang Zheng ${ }^{2}$ and Frederic Kratz ${ }^{1}$
}

\begin{abstract}
This paper focuses on the states estimation and parameter identification for nonlinear Dengue epidemic model. This model supplies a change of coordinates that transforms the studied model into an extended output depending nonlinear observer normal form. Then we can use the high gain observer strategy, which allows both the parameter identification and the state estimation.
\end{abstract}

\section{INTRODUCTION}

The Dengue fever is endemic throughout the tropics and no specific treatment has been developed yet. The disease is transmitted between humans throughout mosquito bites. This spreading process through mosquitoes is given by a nonlinear dynamical system by involving two species (mosquitoes and humans). Each species is partitioned into two populations; the susceptible and the infected one. In this process, the mosquitoes are considered as vectors of disease. In order to minimize its impact on society, numerous studies have been conducted to understand the behavior of dynamics of such diseases which resulted in the development of several models aimed to analyze and predict the evolution of such epidemic in the purpose of minimizing its impact on society.

The modeling of epidemic spread was initiated by [1], that had led to the well-known SIR model (Susceptible, Infected and Removed). Afterwards, it has been improved and adapted according to the type of disease. Based on these models, several works are realized to study the volatility of diseases [2], and to develop vaccination strategy [3]. However, as far as we know, there are few works dedicated to the observer design [4].

To achieve an observer design for the dengue epidemic model, we will use the approach based on nonlinear observer normal forms (NONF). This concept has been set up in [5] for a varying time and in [6] for an invariant-time single output nonlinear dynamical system. Then, it has been adopted for the multiple outputs case in [7], [8]. Afterwards, several algorithms have been developed in [9], [10], [11], [12], [13], [14], [15] and [16]. However, the conditions for the existence of diffeomorphism reported in the above works are relatively too restrictive. Therefore, to enhance this concept and to expand the class of nonlinear systems that allow a change of coordinates, another interesting form is the so-called depending output observer normal form introduced

*This work was supported by Région Centre France

1 R. Tami (ramdane.tamilinsa-cvl.fr), D. Boutat (driss.boutateinsa-cvl.fr) and F. Kratz (frederic.kratzeinsa-cvl.fr) are with INSA Centre Val de Loire, Univ. d'Orléans, Laboratoire PRISME EA 4229, 88 BD Lahitolle, CS 60013, 18022 Bourges, France.

2 G. Zheng (gang. zheng@inria.fr) is with INRIA Lille-Nord Europe, 40 Avenue Halley 59650, France. by [17], and then improved in [18], [19], [20], [21], [22] and [23]. The most recent normal form, called extended nonlinear observer normal form due to [24], allows to enlarge the class of observer normal forms. It has been generalized by [25], [26], [27], [28] and [29]. The idea of the last form is to add an auxiliary dynamics into the original system such that the augmented system fulfills commutativity condition of Krener and Isidori frame [6], which guarantees a transformation of the studied system into an extended normal form [30], [31]. On the other hand, this observer normal form provides an adaptive observer to resolve the simultaneous parameters identification and state estimation problem [32]. Thus, we believe that its application to epidemics model can constitute a very powerful decision-making tool in the vaccination campaign and fighting against diseases. The objective of this paper is intended to show the effectiveness of the observer design to estimate the spreading disease and to identify the parameters in the epidemic model.

This paper is organized as follows. Section II illustrates the formalization of extended output depending observer normal form. Section III presents the dengue epidemic model. Section IV supplies a change of coordinates allowing the transformation of the dengue epidemic model into an extended output depending normal form. Section V deals with simultaneous state estimation and parameter identification of the studied system.

\section{BACKGROUND ON NONLINEAR EXTENDED OBSERVER NORMAL FORMS}

We Consider a nonlinear system in the following form:

$$
\left\{\begin{array}{l}
\dot{x}=f(x) \\
y=h(x)
\end{array}\right.
$$

where $x \in U \subseteq \mathbb{R}^{n}$ represents the state and $y \in \mathbb{R}^{p}$ denotes the output. We assume that the vector field $f$ and the output function $h$ are sufficiently smooth. In the following, we also assume that the pair $(h, f)$ satisfies the observability rank condition, thus the 1 -forms $\theta_{i}=d L_{f}^{i-1} h$ for $i=1: n$ are linearly independent where $L_{f}^{i-1} h$ is the $(i-1)^{t h}$ Lie derivative of the output $h$ in the direction of the vector field $f$, and $d$ is the usual differential. Under this assumption, theoretically the state can be estimated as $x=\sigma\left(y, \dot{y}, \ldots, y^{n-1}\right)$. However, it has been established that the use of successive output derivatives amplifies the noise in the measurement. Therefore, the observer design is a powerful tool to address the state estimation problem. To do so, we firstly present a class of nonlinear systems for which the design of observer is relatively straightforward. 
Consider the nonlinear system (1), we seek an auxiliary dynamics $\dot{w}=\eta(y, w)$ so that the following extended dynamical system:

$$
\begin{aligned}
\dot{x} & =f(x) \\
\dot{w} & =\eta(y, w) \\
y & =h(x)
\end{aligned}
$$

can be transformed through a change of coordinates $\left(\xi^{T}, \zeta\right)^{T}=\phi(x, w)$ into the following extended output depending observer normal form:

$$
\begin{aligned}
\dot{\xi} & =A(y, w) \xi+B(y, w) \\
\dot{\zeta} & =B_{n+1}(y, w) \\
y & =C \xi
\end{aligned}
$$

where $\xi \in \mathbb{R}^{n}, y \in \mathbb{R}^{p}, \zeta \in \mathbb{R}, w \in \mathbb{R}$ is an extra-output, and

$$
A(y, w)=\left(\begin{array}{ccccc}
0 & \ldots & \ldots & \ldots & 0 \\
\alpha_{2}(y, w) & 0 & \ldots & \ldots & 0 \\
0 & \alpha_{3}(y, w) & \ldots & \ldots & \ldots \\
0 & \ldots & \ldots & \ldots & \ldots \\
0 & \ldots & \ldots & \alpha_{n}(y, w) & 0
\end{array}\right)
$$

where $\alpha_{i}(y, w) \neq 0$ for $i=2: n$ is a function depending only on the output $y$ and the extra-output $w$. The normal form (5-7) has a great interest because it supports Kalmanlike observer or the following high gain observer presented in [33]:

$$
\begin{aligned}
& \dot{\hat{\xi}}=A(y, w) \hat{\xi}+B(w, y)-\Gamma^{-1}(y) R_{\rho}^{-1} C^{T}(C \hat{\xi}-\bar{y})(9) \\
& 0=\rho R_{\rho}+G^{T} R_{\rho}+R_{\rho} G-C^{T} C
\end{aligned}
$$

where

$$
\begin{gathered}
G=\left(\begin{array}{cccc}
0 & \cdots & 0 & 0 \\
1 & \cdots & 0 & 0 \\
\vdots & \ddots & \vdots & \vdots \\
0 & \cdots & 1 & 0
\end{array}\right) \\
\Gamma(y, w)=\operatorname{diag}\left[\prod_{i=2}^{n} \alpha_{i}(y, w), \prod_{i=3}^{n} \alpha_{i}(y, w), \cdots, \alpha_{n}(y, w), 1\right] \\
R_{\rho}(n+1-i, n+1-j)=\frac{(-1)^{i+j} C_{i+j-2}^{j-1}}{\rho^{i+j-1}}
\end{gathered}
$$

for $1 \leq i \leq n$ and $1 \leq j \leq n$.

If we set $e=\hat{\xi}-\xi$ to be the observation error, then we see that its error dynamics is governed by the following equation:

$$
\dot{e}=\dot{\hat{\xi}}-\dot{\xi}=\left(A(y, w)-\Gamma^{-1}(y, w) R_{\rho}^{-1} C^{T} C\right) e .
$$

If $y$ and $w$ are bounded, then the observation error dynamics is exponentially stable by well choosing $\rho$.

\section{Dengue Disease Model}

Dengue is a viral infection transmitted by the bite of mosquito Aedes aegypti to human. It is an endemic in many countries through the world, it has not a specifical treatment and the more adapted solution is the prevention.

Throughout this paper, we consider the dengue epidemic model extracted from [34], [35], whose behavior is governed by a set of differential equations describing epidemic transmission between humans and mosquitoes:

$$
\left\{\begin{array}{l}
\frac{d S(t)}{d t}=b_{1}-\lambda_{1} S V-\mu_{1} S \\
\frac{d I(t)}{d t}=\lambda_{1} S V-\gamma I-\mu_{1} I \\
\frac{d M(t)}{d t}=b_{2}-\lambda_{2} M I-\mu_{2} M \\
\frac{d V(t)}{d t}=\lambda_{2} M I-\mu_{2} V
\end{array}\right.
$$

where $S(t)$ and $I(t)$ are respectively the susceptible and infected individuals in human population, $M(t)$ and $V(t)$ are respectively the susceptible and infected mosquitoes. The involving parameters are the following senses: $b_{1}$ is the rate of natural birth on the host population, $\mu_{1}$ is the natural death rate related to host population, $\lambda_{1}$ is the rate daily biting (or the rate of transmission from $V$ ), $\gamma$ is the rate at which the infected individuals are recovered, $b_{2}$ is the birth rate of vector population (i-e mosquitoes), $\lambda_{2}$ is the transmission rate from $I$ to vectors (or rate daily biting of $I$ ), and $\mu_{2}$ is the natural death rate of mosquitoes (i-e vectors).

Throughout this paper, it is assumed that all information concerning humans data $S(t)$ and $I(t)$ will be available thanks to the health department. Therefore, they are taken as the outputs of the model:

$$
\left\{\begin{array}{l}
h_{1}=y_{1}=S \\
h_{2}=y_{1}=I
\end{array}\right.
$$

Now, we will check the observability rank condition of the above dynamical system (11) with the output defined in (12). For this, let us compute the Lie derivative of the outputs as follows:

$$
\begin{aligned}
\theta_{1}= & d L_{f}^{0} h_{1}=d S \\
\theta_{2}= & d L_{f}^{1} h_{1}=\left(\lambda_{1} V-\mu_{1}\right) d S+\lambda_{1} S d V \\
\theta_{3}= & d L_{f}^{2} h_{1} \\
= & -\left(\lambda_{1} b_{1}-\lambda_{1} \mu_{2} S-2 \mu_{1} \lambda_{1} S-2 \lambda_{1}^{2} S V\right) d V \\
& +\left(\mu_{1}^{2}+\lambda_{1}\left(2 \mu_{1}+\mu_{2}\right) V+\lambda_{1}^{2} V^{2}-\lambda_{1} \lambda_{2} M I\right) d S \\
& -\lambda_{1} \lambda_{2} S I d M-\lambda_{1} \lambda_{2} S M d I \\
\theta_{4}= & d h_{2}=d I
\end{aligned}
$$

We see that $\theta_{i}$ for $1 \leq i \leq 4$ are linearly independent which implies the observability of system (11). Thus, we can estimate the state $V(t)$ and $M(t)$ from the outputs and their derivatives i.e. $(V, M)=\sigma\left(y, \dot{y}, y^{(2)}\right)$.

In order to simplify the calculation, as $I(t)$ and $V(t)$ are measured, then we can remove the dynamics of $I$ in (11). Specifically, we will deal with the following reduced dynamical system:

$$
\left\{\begin{array}{l}
\frac{d S(t)}{d t}=b_{1}-\lambda_{1} S V-\mu_{1} S \\
\frac{d M(t)}{d t}=b_{2}-\lambda_{2} M I-\mu_{2} M \\
\frac{d V(t)}{d t}=\lambda_{2} M I-\mu_{2} V
\end{array}\right.
$$




\section{EXTENDED OUTPUT DEPENDING OBSERVER NORMAL FORM}

This section provides a change of coordinates that transforms the model (13) into (5-7). For this we will process in two steps.

\section{A. First change of coordinates}

This subsection deals with a first transformation that enables to transform the dynamical system (13) into another dynamical system with depending output linear part modulo nonlinear terms which are only function of outputs $I$ and $S$ which will allow, thereafter, an easy use of the extended transformation algorithm.

Proposition 1: The following change of coordinates

$$
\begin{aligned}
z_{3} & =-\frac{1}{\lambda_{1}} \ln (S) \\
z_{2} & =\frac{1}{\lambda_{2}} V-\frac{1}{\lambda_{1}}\left(\frac{\mu_{2}}{\lambda_{2}}+S+I\right) \ln (S)+\frac{S}{\lambda_{1}} \\
z_{1} & =M+V
\end{aligned}
$$

transforms (13) into the following dynamical system

$$
\left\{\begin{array}{l}
\dot{z}_{1}=b_{2}-\mu_{2} z_{1} \\
\dot{z}_{2}=I z_{1}+\beta_{2}(y) \\
\dot{z}_{3}=\lambda_{2} z_{2}+\beta_{3}(y) \\
\widetilde{y}=z_{3}
\end{array}\right.
$$

where

$$
\begin{aligned}
\beta_{3}(y)= & -\lambda_{2}\left(-\frac{\ln (S)}{\lambda_{1}}\left(\frac{\mu_{2}}{\lambda_{2}}+S+I\right)+\frac{S}{\lambda_{1}}\right) \\
& -\frac{b_{1}}{\lambda_{1} S}+\frac{\mu_{1}}{\lambda_{1}} \\
\beta_{2}(y)= & \left(-\frac{b_{1}}{\lambda_{1} S}+\frac{\mu_{1}}{\lambda_{1}}\right)\left(S+I+\frac{\mu_{2}}{\lambda_{2}}\right) \\
& -\frac{\ln (S)}{\lambda_{1}}\left(b_{1}-\mu_{1} S-\left(\gamma+\mu_{1}\right) I\right) \\
& +\frac{\left(b_{1}-\mu_{1} S\right)}{\lambda_{1}}
\end{aligned}
$$

Proof: A direct derivation of change of coordinates (16) implies $\dot{z}_{3}=-\frac{\dot{S}}{\lambda_{1} S}$. Now, from the model (11), we obtain:

$$
\begin{aligned}
\dot{z}_{3}= & \lambda_{2} z_{2}-\lambda_{2}\left(-\frac{\ln (S)}{\lambda_{1}}\left(\frac{\mu_{2}}{\lambda_{2}}+S+I\right)+\frac{S}{\lambda_{1}}\right) \\
& -\frac{b_{1}}{\lambda_{1} S}+\frac{\mu_{1}}{\lambda_{1}}
\end{aligned}
$$

which can be rewritten into the following compact form:

$$
\dot{z}_{3}=\lambda_{2} z_{2}+\beta_{3}(y) \text {. }
$$

In the same way, the derivative of the second component of change of coordinates (15) gives:

$$
\begin{aligned}
\dot{z}_{2}= & \frac{1}{\lambda_{2}} \dot{V}+\mu_{2} \dot{z}_{3}+\dot{z}_{3}(S+I)+z_{3}(\dot{S}+\dot{I})+\frac{1}{\lambda_{1}} \dot{S} \\
= & I z_{1}+\left(-\frac{b_{1}}{\lambda_{1} S}+\frac{\mu_{1}}{\lambda_{1}}\right)\left(S+I+\frac{\mu_{2}}{\lambda_{2}}\right) \\
& -\frac{\ln (S)}{\lambda_{1}}\left(b_{1}-\mu_{1} S-\left(\gamma+\mu_{1}\right) I\right)+\frac{\left(b_{1}-\mu_{1} S\right)}{\lambda_{1}}
\end{aligned}
$$

Therefore, we obtain the second differential equation in Proposition 1 as follows:

$$
\dot{z}_{2}=I z_{1}+\beta_{2}(y) .
$$

For the third component of the change of coordinates (16), a straightforward calculation leads to

$$
\dot{z}_{1}=\dot{M}+\dot{V}=b_{2}-\mu_{2} z_{1}
$$

However, due to the first dynamics in system (17), one can see that it is different from the proposed observer normal form (5)-(7).And this motivates us to introduce the concept of extended dynamics (or immersion) to overcome this limitation.

\section{B. Extended Dynamics}

In this subsection, we will use the concept of extended dynamics. For this, let us extend the dynamical system (17) as follows

$$
\left\{\begin{array}{l}
\dot{z}_{1}=b_{2}-\mu_{2} z_{1} \\
\dot{z}_{2}=I z_{1}+\beta_{2}(y) \\
\dot{z}_{3}=\lambda_{2} z_{2}+\beta_{3}(y) \\
\dot{w}=\eta(y, w)
\end{array}\right.
$$

where $w \in \mathbb{R}$ is an auxiliary variable considered as an extra output and $\eta(y, w)$ is a function to be determined ( for more details see [36], [24], [26], [27] and [28]).

Proposition 2: The following change of coordinates

$$
\begin{aligned}
& \xi_{1}=l(w) z_{1} \\
& \xi_{2}=z_{2}, \quad \xi_{3}=z_{3}, \quad \xi_{4}=w
\end{aligned}
$$

transforms the dynamical system (18) into the following extended nonlinear output depending observer normal form

$$
\left\{\begin{array}{l}
\dot{\xi}_{1}=B_{1}(y, w) \\
\dot{\xi}_{2}=\frac{I}{l(w)} \xi_{1}+B_{2}(y, w) \\
\dot{\xi}_{3}=\lambda_{2} \xi_{2}+B_{3}(y, w) \\
\dot{\xi}_{4}=\eta(y, w) \\
y=S
\end{array}\right.
$$

where $\eta(w)$ is only function of $w$ and $l(w)=e^{\int_{0}^{w} \frac{\mu_{2}}{\eta(s)}}$.

Proof: Firstly, for a given $\eta(y, w) \neq 0$, let us firstly seek the function $l(w)$. A direct derivative of the change of coordinates (19) implies

$$
\dot{\xi}_{1}=z_{1} l^{\prime}(w) \dot{w}+l(w) \dot{z}_{1} .
$$

Now, from the first and the fourth differential equations of (18), we obtain

$$
\dot{\xi}_{1}=z_{1} l^{\prime}(w) \eta(y, w)-l(w) \mu_{2} z_{1}+l(w) b_{2} .
$$

As intended by observer normal form (21), the dynamics $\dot{\xi}_{1}$ should be a function of the output $(S, I, w)$, therefore, we require that

$$
z_{1} l^{\prime}(w) \eta(y, w)-l(w) \mu_{2} z_{1}=0
$$

which leads to the following differential equation

$$
\frac{l^{\prime}(w)}{l(w)}=\frac{\mu_{2}}{\eta(y, w)} .
$$


As $l(w)$ is chosen as only a function of $w$, then from the last equation (22), the function $\eta(w, y)=\eta(w)$ must be also only a function of $w$.

Then, by integrating of equation (22) we obtain

$$
l(w)=e^{\int_{0}^{w} \frac{\mu_{2}}{\eta(s)} d s} .
$$

Therefore, the dynamics of $\xi_{1}$ becomes

$$
\dot{\xi}_{1}=B_{1}(y, w)=l(w) b_{2} .
$$

Now, taking into account the dynamical system (18), the derivative of the second variable (20) implies:

$$
\begin{aligned}
\dot{\xi}_{2}= & \frac{I}{l(w)} \xi_{1}+\left(-\frac{b_{1}}{\lambda_{1} S}+\frac{\mu_{1}}{\lambda_{1}}\right)\left(S+I+\frac{\mu_{2}}{\lambda_{2}}\right) \\
& -\frac{1}{\lambda_{1}}\left(b_{1}-\mu_{1} S-\left(\gamma+\mu_{1}\right) I\right)(\ln (S)) \\
& +\frac{1}{\lambda_{1}}\left(b_{1}-\mu_{1} S\right)
\end{aligned}
$$

which can be written as follows:

$$
\dot{\xi}_{2}=\alpha_{2}(y, w) \xi_{1}+B_{2}(y, w) .
$$

In the same way, the derivative of $\xi_{3}$ leads to

$$
\begin{aligned}
\dot{\xi}_{3}= & \lambda_{2} \xi_{2}-\lambda_{2}\left(-\frac{1}{\lambda_{1}} \ln (S)\left(\frac{\mu_{2}}{\lambda_{2}}+S+I\right)+\frac{1}{\lambda_{1}} S\right) \\
& -\frac{b_{1}}{\lambda_{1} S}+\frac{\mu_{1}}{\lambda_{1}}
\end{aligned}
$$

and this can be described into the following compact form

$$
\dot{\xi}_{3}=\alpha_{3}(y, w) \xi_{2}+B_{2}(y, w)
$$

and finally the dynamics of $\xi_{4}$ is given by

$$
\dot{\xi}_{4}=\eta(y, w)=B_{4}(y, w) .
$$

\section{Simulation results}

For simulation, we use the following parameters: $b 1=$ 0.01, $\lambda_{1}=5 E-4, \mu_{1}=0.01, \gamma=0.01, \lambda_{2}=3 E-4: 10$, $b_{2}=60 ; \mu_{2}=0.5$. The initial conditions are $S(0)=784$, $I(0)=216, R(0)=0 ; M(0)=0, V(0)=100$. To ensure the boundedness of $w$, we choose $\dot{w}=\eta(w)=\frac{\sin a w}{a w} \mu_{2}$ with $a=0.0005$. The simulation results are presented in Fig (1-3)

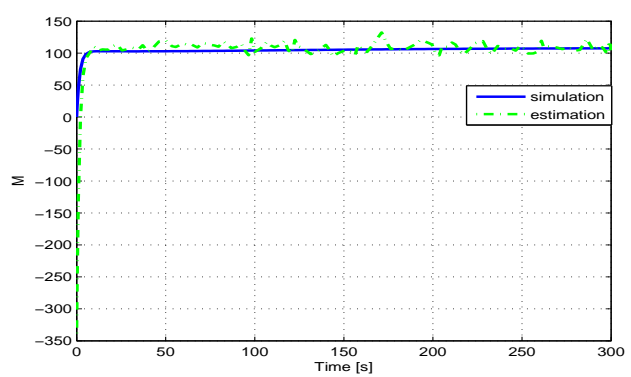

Fig. 1. Evolution of population $(M)$

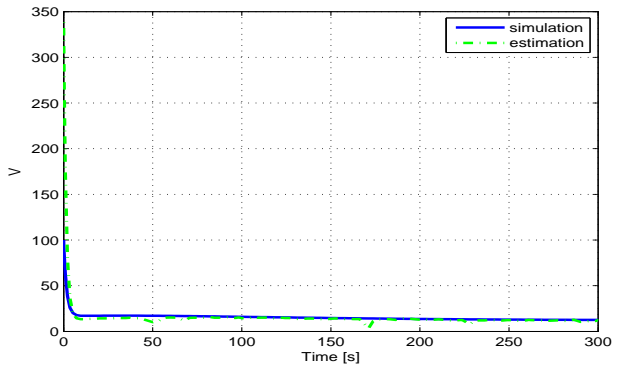

Fig. 2. Evolution of population $(V)$

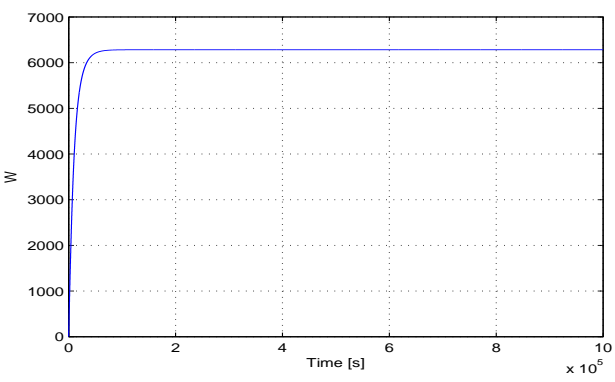

Fig. 3. Boundedness of auxiliary dynamics $(w)$

\section{AdAPtive observer}

Unlike the above section, this section deals with simultaneous parameter identification and state estimation for system (13). It assumes that the parameters $b_{2}, \mu_{2}, \gamma_{2}$ of the second and the third equation in system (13) are unknown. Therefore, inspired by the works of [32], [37] and [38], we rewrite system (21) into the following affine unknown parameters observer normal form:

$$
\left\{\begin{array}{l}
\dot{\xi}=A(w, y) \xi+\varphi(y)+\phi(w, y) \theta \\
y=C \xi
\end{array}\right.
$$

where $\xi=\left[\begin{array}{lll}\xi_{1} & \xi_{2} & \xi_{3}\end{array}\right]^{T}, \theta=\left[\begin{array}{llll}b_{2} & \frac{\mu_{2}}{\lambda_{2}} & \mu_{2} & \lambda_{2}\end{array}\right]^{T}$

$$
\begin{gathered}
A(w, y)=\left[\begin{array}{ccc}
0 & 0 & 0 \\
\alpha_{2}(w, y) & 0 & 0 \\
0 & \alpha_{3} & 0
\end{array}\right]=\left[\begin{array}{ccc}
0 & 0 & 0 \\
\frac{I}{l(w)} & 0 & 0 \\
0 & \lambda_{2} & 0
\end{array}\right] \\
\phi(w, y)=\left[\begin{array}{cccc}
l(w) & 0 & 0 & 0 \\
0 & \frac{\mu_{1} S-b_{1}}{\lambda_{1} S} & 0 & 0 \\
0 & 0 & \frac{\ln (S)}{\lambda_{1}} & \frac{(S+I) \ln (S)+S}{\lambda_{1}}
\end{array}\right]
\end{gathered}
$$

and

$$
\varphi(y)=\left[\begin{array}{c}
0 \\
\left(\frac{\mu_{1} S-b_{1}}{\lambda_{1} S}\right) I-\left(b_{1}-\mu_{1} S-\left(\gamma+\mu_{1}\right) I\right) \frac{\ln (S)}{\lambda_{1}} \\
\frac{\mu_{1}}{\lambda_{1}}-\frac{b_{1}}{\lambda_{1} S}
\end{array}\right]
$$

Similarly to [38] and [37], it is assumed as well that there exist positive constants $\alpha, \beta, T$ such that

$$
\alpha I \leq \int_{t}^{t+T} \Lambda^{T}(\tau) C^{T} \Sigma(\tau) C(\tau) \Lambda(\tau) d r \leq \beta I
$$


and

$$
\alpha I \leq \int_{t}^{t+T} \Psi(t, \tau)^{T} C^{T} \Sigma(\tau) C \Psi(t, \tau) d r \leq \beta I \quad \forall t \geq t_{0}
$$

where $\Psi$ is the transition matrix for the system

$$
\left\{\begin{array}{l}
\dot{x}=A(y, w) x \\
y=C x
\end{array}\right.
$$

and $\Sigma$ is a positive definite bounded matrix. Then, based on the results in [32], [37] and [38], the following system:

$$
\begin{aligned}
\dot{\hat{x}}= & A(y, w) \widehat{x}+\varphi(y)+\phi(y, w) \widehat{\theta} \\
& +\left\{\Lambda S_{\theta}^{-1} \Lambda^{T} C^{T}+S_{x}^{-1} C^{T}\right\} \Sigma(y-C \widehat{x}) \\
\dot{\hat{\theta}}= & S_{\theta}^{-1} \Lambda^{T} C^{T} \Sigma(y-C \widehat{x}) \\
\dot{\Lambda}= & \left\{A(y, w)-S_{x}^{-1} C^{T} C\right\} \Lambda+\phi(y, w) \\
\dot{S}_{x}= & -\rho_{x} S_{x}-A(y, w)^{T} S_{x}-S_{x} A(y, w)+C^{T} \Sigma(2) \\
\dot{S}_{\theta}= & -\rho_{\theta} S_{\theta}+\Lambda^{T} C^{T} \Sigma C \Lambda, \quad S_{x}(0), S_{\theta}(0)>0
\end{aligned}
$$

where $\rho_{x}$ and $\rho_{\theta}$ are positive constants, is an exponential adaptive observer for the nonlinear system (23).

Remark 3: According to [33], as we have the form (24), then (28) can be simplified to algebraic equation (10). In this case, it implies the following equation:

$$
R_{\rho}=\left(\begin{array}{ccc}
\frac{6}{\rho_{x}^{5}} & \frac{-3}{\rho_{x}^{4}} & \frac{1}{\rho_{x}^{3}} \\
\frac{-3}{\rho_{x}^{4}} & \frac{2}{\rho_{x}^{3}} & \frac{-1}{\rho_{x}^{2}} \\
\frac{1}{\rho_{x}^{3}} & \frac{-1}{\rho_{x}^{2}} & \frac{1}{\rho_{x}}
\end{array}\right)
$$

\section{A. Simulation results}

The figures (4)-(8) present the simulation results obtained with adaptive observer where $\rho_{x}=0.77, \rho_{\theta}=0.05, \Sigma=1$ and the initial conditions are the same as before. The fast convergences show the efficiency of the used approach to estimate the states $(M, V)$ and to identify the parameters $\left(b_{2}, \lambda_{2}, \mu_{2}\right)$.

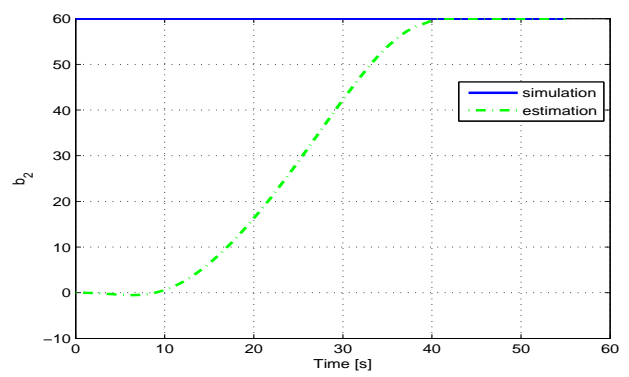

Fig. 4. Identification of parameter $b_{2}$

\section{CONCLUSIONS AND FUTURE WORKS}

The observer design for simultaneous states estimation and parameter identification of dengue epidemic model is studied. The efficiency of the proposed approach mixing the change of coordinates and extended dynamics to construct extended observer normal form is illustrated by the application to practical example.

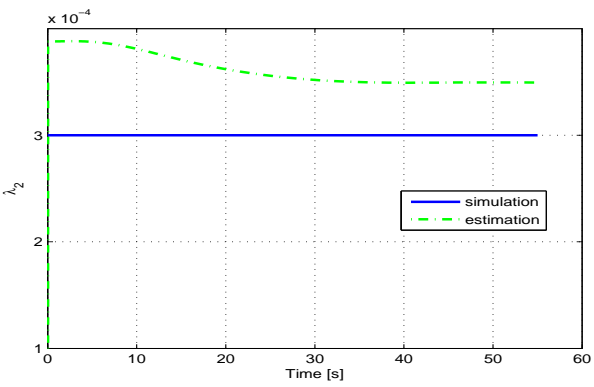

Fig. 5. Identification of parameter $\lambda_{2}$

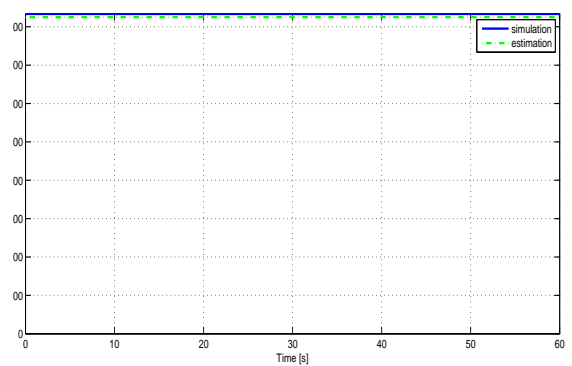

Fig. 6. Identification of parameter $\mu_{2} / \lambda_{2}$

\section{REFERENCES}

[1] W. Kermack and A. McKendrick, "A contribution to the mathematical theory of epidemics," Proc. Royal Soc. London, vol. 115, pp. 700-721, 1927.

[2] M. Fan, M. Y. Li, and K. Wang, "Global stability of an seis epidemic model with recruitment and a varying total population size," Mathematical Biosciences, vol. 170, no. 2, pp. 199 - 208, 2001.

[3] M. De la Sen and S. Alonso-Quesada, "Vaccination strategies based on feedback control techniques for a general seir-epidemic model," Applied Mathematics and Computation, vol. 218, no. 7, p. 38883904 , 2011.

[4] O. Bernard, G. Sallet, and A. Sciandra, "Nonlinear observers for a class of biological systems: application to validation of a phytoplanktonic growth model," IEEE Transactions on Automatic Control, vol. 43 , no. 8 , pp. 1056-1065, 1998.

[5] D. Bestle and M. Zeitz, "Canonical form observer design for nonlinear time varying systems," Internatio, vol. 38, pp. 419-431, 1983.

[6] A. Krener and A. Isidori, "Linearization by output injection and nonlinear observers," Systems \& Control Letters, vol. 3, no. 1, pp. 47-52, 1983.

[7] A. Krener and W. Respondek, "Nonlinear observers with linearizable error dynamics," SIAM Journal on Control and Optimization, , vol. 23, no. 2, pp. 197-216, 1985.

[8] X. Xia and W. Gao, "Nonlinear observer with linearizable error dynamics," SIAM Journalon Control and Optimization,, vol. 27, pp. 199-216, 1989.

[9] H. Keller, "Nonlinear observer design by transformation into a generalized observer canonical form," International Journal of Control, vol. 46(6), pp. 1915-1930, 1987.

[10] A. Phelps, "On constructing nonlinear observers," SIAM Journal on Control and Optimmisation, vol. 29, pp. 516-534, 1991.

[11] J. Rudolph and M. Zeitz, "A block triangular nonlinear observer normal form," Systems \& Control Letters, vol. 23, no. 1, pp. 1-8, 1994.

[12] M. Hou and A. Pugh, "Observer with linear error dynamics for nonlinear multi-output systems," Systems \& Control Letters, vol. 37, pp. 1-9, 1999.

[13] R. Marino and P. Tomei, Nonlinear control design: geometric, adaptive and robust, P. H. I. U. Ltd, Ed., 1996.

[14] D. Boutat, A. Benali, H. Hammouri, and K. Busawon, "New algorithm 


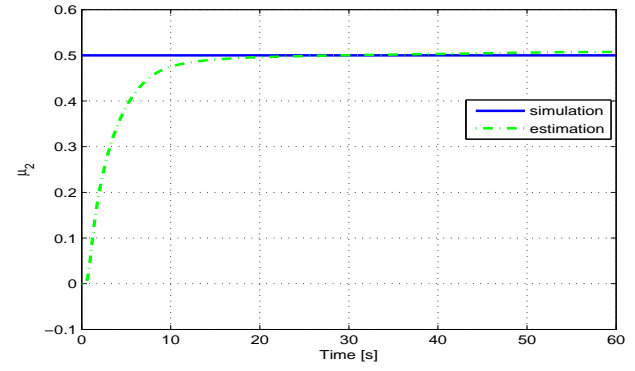

Fig. 7. Identification of parameter $\mu_{2}$
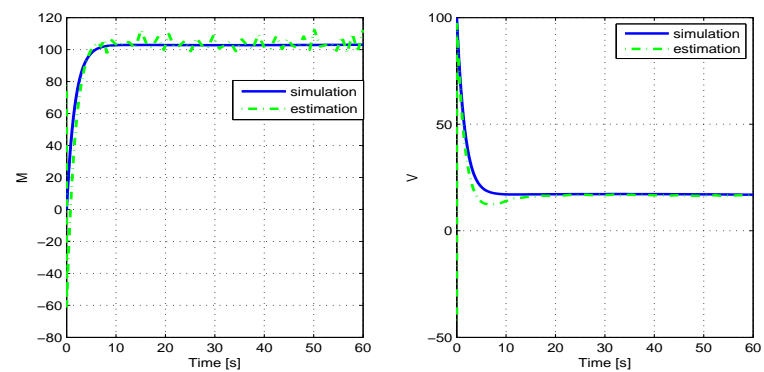

Fig. 8. Estimation of $M$ and $V$

for observer error linearization with a diffeomorphism on the outputs," Automatica, vol. 45, no. 10, pp. 2187-2193, 2009.

[15] A. Lynch and S. Bortoff, "Nonlinear observers with approximately linear error dynamics: the multivariable case," IEEE Transactions on Automatic Control,, vol. 46, no. 6, pp. 927-932, jun 2001.

[16] M. Lopez, F. Plestan, and A. Glumineau, "Linearization by completely generalized input ouput injection,” Kybernetika,, vol. 35, pp. 793-802, 1999.

[17] W. Respondek, A. Pogromsky, and H. Nijmeijer, "Time scaling for observer design with linearizable error dynamics," Automatica, vol. 40 (2), pp. 277-285, 2004.

[18] G. Zheng, D. Boutat, and J. Barbot, "A single output dependent observability normal form," SIAM Journal on Control and Optimization, vol. 46, no. 6, pp. 2242-2255, 2007.

[19] G. Zheng and D. Boutat, "Synchronisation of chaotic systems via reduced observers," IET Control Theory \& Applications, vol. 5, no. 2, pp. 308-314, 2011.

[20] D. Boutat, G. Zheng, J.-P. Barbot, and H. Hammouri, "Observer error linearization multi-output depending," IEEE CDC, 2006.

[21] G. Zheng, D. Boutat, and J.-P. Barbot, "Multi-output dependent observability normal form," Nonlinear Analysis: Theory, Methods \& Applications, vol. 70, no. 70, pp. 404-418, 2009.

[22] - "Output dependent observability linear normal form," IEEE $C D C-E C C, 2005$.

[23] Y. Wang and A. Lynch, "Observer design using a generalized timescaled block triangular observer form," Systems \& Control Letters, vol. 58, pp. 346-352, 2009.

[24] P. Jouan, "Immersion of nonlinear systems into linear systems modulo output injection," SIAM Journal on Control and Optimisation,, vol. 41(6), pp. 1756-1778, 2003.

[25] D. Noh, N. Jo, and J. Seo, "Nonlinear observer design by dynamic observer error linearization," IEEE Transactions on Automatic Control, vol. 49, no. 10, pp. 1746-1753, 2004.

[26] J. Back, K. T. Yu, and J. H. Seo, "Dynamic observer error linearization," Automatica, vol. 42, no. 12, pp. 2195-2200, 2006.

[27] D. Boutat, "Geometrical conditions for observer error linearization via $\int 0,1, \ldots,(n-2)$, , in 7 th IFAC Symposium on Nonlinear Control Systems Nolcos'07,, 2007.

[28] D. Boutat and K. Busawon, "On the transformation of nonlinear dynamical systems into the extended nonlinear observable canonical form," International Journal of Control,, vol. 84, no. 1, pp. 94-106, 2011.
[29] J. Yang, J. Back, J. Seo, and H. Shim, "Reduced-order dynamic observer error linearization," in Nonlinear Control Systems, , 2010, pp. 915-920.

[30] R. Tami, D. Boutat, and G. Zheng, "Extended output depending normal form," Automatica, vol. 49, no. 7, pp. 2192-2198, 2013.

[31] D. Boutat, "Extended nonlinear observer normal forms for a class of nonlinear dynamical systems," International Journal of Robust and Nonlinear Control, pp. n/a-n/a, 2013.

[32] R. Marino and P. Tomei, "Global adaptive observers for nonlinear systems via filtered transformations," IEEE Transactions on Automatic Control, vol. 37, no. 8, pp. 1239-1245, 1992.

[33] K. Busawon, M. Farza, and H. Hammouri, "Asimple observer for a class of nonlinear systems," Applied Mathematics Letters, vol. 11, no. 3, pp. 27-31, 1998 .

[34] L. Esteva and C. Vargas, "Analysis of a dengue disease transmission model," Mathematical Biosciences, vol. 150, no. 2, pp. 131-151, 1998.

[35] Y.-H. Hsieh and C. Chen, "Turning points, reproduction number, and impact of climatological events for multi-wave dengue outbreaks," Tropical Medicine \& International Health, vol. 14, no. 6, pp. 628638, 2009.

[36] M. Guay, "Observer linearization by output-dependent time-scale transformations," IEEE Transactions on Automatic Control,, vol. 47, no. 10 , pp. $1730-1735$, oct 2002 .

[37] Q. Zhang, "Adaptive observer for multiple-input-multiple-output (mimo) linear time-varying systems," IEEE Transactions on Automatic Control, vol. 47, no. 3, pp. 525-529, 2002.

[38] G. Besançon, J. de Leon-Morales, and O. Huerta-Guevara, "On adaptive observers for state affine systems and application to synchronous machines," in 42nd IEEE Conference on Decision and Control. Proceedings., vol. 3. IEEE, 2003, pp. 2192-2197. 